Jing Wang* and Sheila N. Baker

\title{
Pyrrolidinium salt based binary and ternary deep eutectic solvents: green preparations and physiochemical property characterizations
}

https://doi.org/10.1515/gps-2017-0060

Received April 19, 2017; accepted June 9, 2017; previously published online August 25, 2017

\begin{abstract}
Ionic liquids (ILs) are considered to be green solvents for various applications. However, their synthesis via chemical reaction with by-products or waste produced is contradictory to the concept of green chemistry, and the purity problem and economic feasibility limit their applications in some large-scale industrial applications. 1-Butyl-1-methylpyrrolidinium bromide ([bmpy][Br]), which is a molten salt with melting point above $100^{\circ} \mathrm{C}$ is a precursor of pyrrolidinium ILs, but hardly can be put under the category of IL because of its high melting point. In this study, [bmpy][Br] based binary deep eutectic solvent (BDES) and ternary deep eutectic solvent (TDES) were synthesized to prepare [bmpy] $[\mathrm{Br}]$ in liquid form. During the preparation process, no reaction media was employed, no by-product was generated, and no further purification was required, thereby making it a completely green process. The prepared TDES has better thermal stability and larger free volume than BDES, which is potentially useful for sorption applications with high temperature requirement. It is also because of the green preparation process that the TDES is also expected to be capable for the largescale industrial applications. This work is opening up new avenues for the study of binary and ternary IL-DES system and their applications.
\end{abstract}

Keywords: binary and ternary mixtures; deep eutectic solvent; green chemistry; ionic liquid.

\section{Introduction}

Ionic liquids (ILs) have attracted great interest as environmentally responsible designer solvents compared to the volatile organics because of their featured properties

*Corresponding author: Jing Wang, Department of Mechanical and Aerospace Engineering, University of Missouri-Columbia, Columbia, M0 65211, USA, e-mail: wajing@missouri.edu

Sheila N. Baker: Department of Chemistry, University of MissouriColumbia, Columbia, MO 65211, USA like high viscosity, low vapor pressure and thermal stability [1]. However, the synthesis of ILs by chemical reaction has high requirements with regard to temperature and pressure, needs reaction media, generates by-product and wastes, which makes them hardly to be called as green solvents from the view of synthesis and processing [2]. Furthermore, although some ILs might have good performance in lab-scale applications, their high prices limit their applications in large-scale industrial applications [3].

It has been more than a decade since the introduction of the term deep eutectic solvent (DES) [4], but it has become a hot topic only since the last few years. DES is usually formed between halide salts and hydrogen bond donors (e.g. alcohol, acid) leading to a mixture with lower freezing/melting point than the single components. Therefore, DES is sometimes called low-transition-temperature mixtures (LTTMs) [5]. DES has physiochemical properties similar to the common ILs, so it is considered as an alternative for ILs [2]. Scientist have utilized DESs in a similar way to ILs in many applications [6,7]. Compared with IL, DES has certain advantages like simple preparation, better economic feasibility and low toxicity [8], which make it more suitable for the large-scale industrial applications. With the concept of DES, researchers have successfully utilized it for applications in the area of separation processes [9-12], nanomaterials [13], electrochemistry [14], polymer science [15, 16], bioengineering [17-19], and organic synthesis [20-22]. DES is playing an increasingly important role in green chemistry and processing [23, 24]. Extensive experiments are ongoing to study the properties of novel synthesized DES [25-27], and they have provided solid foundation for the potential applications which can take advantages of both ILs and DES.

One property of ILs that contributes to the diversity of IL functions is the designable property of ILs by combining different cations and anions; it can also be applied to DES [28]. Thus DES is also designable by changing one or both the components or some functional groups in the components to achieve the desired function [29]. The conventional DES is formed from two single components, and is of binary deep eutectic solvent (BDES). Compared with the traditional BDES, the introduction of a third component in the ternary deep eutectic solvent (TDES) further enhanced 
the designable property. To date, TDES has been synthesized and applied for various applications [30-32]. The emergence of the terms DES-based IL [33] or IL-based DES [34] indicated researchers' expectation that the combined properties of ILs and DES are in need. ILs are defined as the molten salts with melting points below $100^{\circ} \mathrm{C}$, so some salts do not come under the category of IL because of their high melting points despite the similar structures [35]. 1-Butyl-1-methylpyrrolidinium bromide ([bmpy][Br] $)$ is an example, which could be transferred to be IL by an anion exchange reaction [36]. A dilemma comes to this kind of salts: if changing the anion, it is easy to bring the purity problem because of the chemical reaction; if retaining the anion, the solid state of the salt limits the applications like IL. One of the advantages of preparing DES is to lower the freezing/melting point of the single component, further eliminating the dilemma of some ILs referred earlier. Furthermore, the IL-DES ionic fluids provide tunable moieties as ILs, and can also provide tunable property possibilities. Synthesizing the molten salt into DES will not only expand avenues for the applications of DES, but also provide more opportunities for both IL and DES development.

In this study, we focused on the pyrrolidinium molten salt [bmpy] [Br]. The synthesis of DES usually starts with salt with quaternary ammonium cation and halide anion interacting with hydrogen bond donors. The structure of the pyrrolidinium ring (Figure 1) could be regarded as a quaternary ammonium structure with two side chains connected, which is similar to the precursor of a DES. Glycerol (Gly) is a commonly used renewable solvent in a variety of applications [37], and is also a commonly used hydrogen bond donor for DES preparations [38]. Compared with other widely studied IL categories like imidazolium ILs, pyrrolidinium ILs usually have lower viscosity and less toxicity [39], which enables the [bmpy][Br] based IL-DES ionic fluids to have more potential combined properties of ILs and DES. In this study, the tested thermal properties of ionic fluids are useful in the settings of

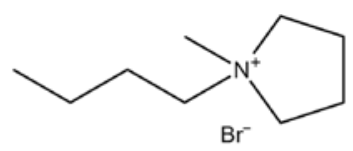

1-Butyl-1-methylpyrrolidinium bromide ([bmpy][Br] $)$

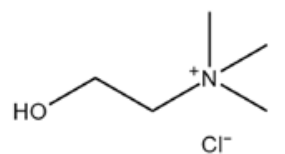

Choline chloride $(\mathrm{ChCl})$

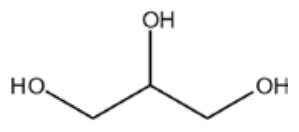

Glycerol (Gly)
Figure 1: Chemical structures of [bmpy][Br], choline chloride and glycerol. process operation conditions, while the measured physical properties are useful for the design of the contacting equipment and modeling of the process [31].

\section{Materials and methods}

\subsection{Reagents and materials}

Choline chloride ( $\mathrm{ChCl},>99 \%$ ) was purchased from Chem-Impex International Inc. (Wood Dale, USA). Glycerol (Gly, >99.5\%), 1-bromobutane (99\%), 1-methylpyrrolidine $(\geq 98 \%)$ and diethyl ether $(\geq 99.7 \%)$ were purchased from Sigma-Aldrich (St. Louis, USA). All chemicals were used as purchased.

\subsection{Synthesis and preparation protocols}

([bmpy][Br]) was synthesized by using the previously reported method [36]. In short, 1-bromobutane was added into 1-methylpyrrolidine to react for 1 day in cooling bath, purified with diethyl ether and water, and decolorized with charcoal. After lyophilization, pure white solid of [bmpy][Br] was obtained. The chemical structures of $\mathrm{ChCl}$, Gly and [bmpy][Br] are shown in Figure 1. BDES of [bmpy] $[\mathrm{Br}]$ and Gly were prepared by mixing the two components at molar ratios from 1:1 to $1: 3$. By heating the binary mixture to $80^{\circ} \mathrm{C}$ with stir, clear liquids were obtained. After cooling to room temperature, the binary mixture remains at liquid state. $\mathrm{DES}(1)$ of $\mathrm{ChCl}$ and $\mathrm{Gly}$ was prepared by stirring the two components at a molar ratio of 1:2 (ChCl:Gly) at $80^{\circ} \mathrm{C}$ as reported [40]. TDES of [bmpy] [Br], $\mathrm{ChCl}$ and Gly ended up with clear liquids were prepared by mixing different mass of [bmpy] $[\mathrm{Br}]$ into DES(1) at ratios from 1:0.5 to 1:5 at $80^{\circ} \mathrm{C}$. After cooling to room temperature, the ternary mixture also remains in liquid state.

\subsection{Instrumentation}

Thermal analysis was conducted with thermogravimetric analysis (TGA) and differential scanning calorimetry (DSC). TGA was performed using a model TGA Q500 (TA Instruments, Inc., New Castle, USA) at a ramping rate of $10^{\circ} \mathrm{C} \mathrm{min}-1$ from room temperature to $600^{\circ} \mathrm{C}$ under nitrogen. DSC was performed using a model DSC Q20 (TA Instruments, Inc., New Castle, USA) at a ramping rate of $2^{\circ} \mathrm{C} \mathrm{min}^{-1}$ under nitrogen. Viscosities at different temperature were obtained using a Brookfield DV-III Ultra Programmable Rheometer with Spindle CPE-42 (AMETEK Brookfield, Middleboro, USA) and equipped with a Fisher Scientific Isotemp ${ }^{\circledR}$ temperature bath (Thermo Fisher Scientific, Waltham, USA). Densities were measured at room temperature $\left(22^{\circ} \mathrm{C}\right)$ by weighing in a standard $2.0 \mathrm{~cm}^{-3}$ specific gravity bottle. At least three readings were collected for analysis.

\section{Results and discussion}

For the synthesis process of either the BDES or TDES in this study, it is notable that no chemical reaction media 

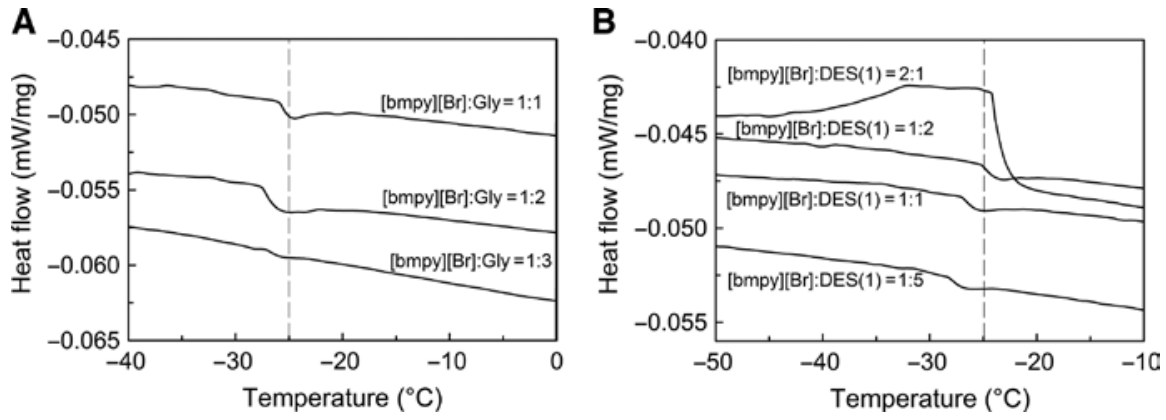

Figure 2: DSC curves of (A) BDES and (B) TDES taken from the second cooling cycle. The BDES are composed of [bmpy][Br] and glycerol in different molar ratios and the TDES is composed of [bmpy] $[\mathrm{Br}]$ and $\mathrm{DES}(1)(\mathrm{ChCl}: \mathrm{Gly}=1: 2$ molar ratio) in different mass ratios, as shown in the figure.

or solvent was needed, no by-product was generated, and no purification step was required. The synthesis process was simply heating and mixing. As the heating temperature was not high $\left(80^{\circ} \mathrm{C}\right)$, and the mixing time was short $(\sim 20 \mathrm{~min})$, the process was very energy saving. The whole process was $100 \%$ atom conversion and completely met the requirements of green chemistry.

\subsection{Phase behavior}

The prepared mixtures were in liquid form when mixing at $80^{\circ} \mathrm{C}$. After cooling to room temperature, the mixtures still remained at liquid state instantly. After setting steady for a week, all prepared mixtures were clear liquid without separation of phases or sediment except for [bmpy][Br]:DES(1)=2:1 becoming solidified and cloudy white. This phenomenon indicated that when the mixtures were prepared close to the eutectic ratios, the prepared DESs are expected to be steady at room temperature without change of phases.

\subsection{Thermal properties}

The DSC results for the [bmpy][Br] and Gly BDES and [bmpy][Br] and DES(1) TDES are shown in Figure 2, which were taken from the second cooling cycles of the DSC runs (Figure S1-Figure S7 in Supplementary Material). There were no major peaks (e.g. melting or crystallizing peak) observed except for a stage at around $-25^{\circ} \mathrm{C}$ for each sample, which is the glass transition stage. $T_{\mathrm{g}}$ was determined by the midpoint method, the middle of the step measured as half the step height. A summary of $T_{\mathrm{g}}$ is shown in Table 1. As the temperature differences were within $3^{\circ} \mathrm{C}$, which could be considered as errors, there were no statistically significant temperature differences among the DES in different ratios. However, in the DSC curves of [bmpy] $[\mathrm{Br}]$ (Figure S8), there were endothermal peak events observed. The disappearance of the endothermal peaks indicated the formation of the hydrogen bonding interactions. In some cases, an endothermal peak with an onset temperature recognized as the eutectic temperature could be observed in the DSC curves. Morrison et al. [41] observed an endotherm onset temperature of $17^{\circ} \mathrm{C}$ in the $\mathrm{DSC}$ curve of $\mathrm{ChCl}$ and urea at 1:2 molar ratio, which is similar to the previously reported data by naked eyes observation [42]. However, the DSC curves of malonic acid and $\mathrm{ChCl}$ eutectic mixture showed no thermal events except for the shift in base line at above $-50^{\circ} \mathrm{C}$ [41]. The lack of the nucleation of malonic acid and $\mathrm{ChCl}$ system was considered to prohibit the appearance of the expected peak events. More examples with $T_{\mathrm{g}}$ without melting peaks in the DSC curves were shown in a mini review by Francisco et al. [5]. For the liquid mixtures with no melting peaks observed but lower $T_{\mathrm{g}}$ obtained, Francisco et al. [43] also suggested to name them as LTTMs instead of DESs.

The thermal stability of the DES was studied based on the TGA results, which are shown in Figure 3. There was only one single decreasing stage for each sample, which

Table 1: Glass transition temperature $\left(T_{\mathrm{g}}\right)$ of the prepared DES.

\begin{tabular}{lc}
\hline DES ratio $^{\mathrm{a}}$ & $\boldsymbol{T}_{\mathrm{g}}\left({ }^{\circ} \mathrm{C}\right)$ \\
\hline$[\mathrm{bmpy}][\mathrm{Br}]: \mathrm{Gly}=1: 1$ & -25.5 \\
{$[\mathrm{bmpy}][\mathrm{Br}]: \mathrm{Gly}=1: 2$} & -26.5 \\
{$[\mathrm{bmpy}][\mathrm{Br}]: \mathrm{Gly}=1: 3$} & -26.0 \\
{$[\mathrm{bmpy}][\mathrm{Br}]: \mathrm{DES}(1)=2: 1$} & -24.0 \\
{$[\mathrm{bmpy}][\mathrm{Br}]: \mathrm{DES}(1)=1: 1$} & -26.0 \\
{$[\mathrm{bmpy}][\mathrm{Br}]: \mathrm{DES}(1)=1: 2$} & -24.5 \\
{$[$ bmpy][Br]:DES$(1)=1: 5$} & -27.0 \\
\hline
\end{tabular}

aThe BDES is composed of [bmpy][Br] and glycerol in different molar ratios. The TDES is composed of [bmpy] $[\mathrm{Br}]$ and $\mathrm{DES}(1)(\mathrm{ChCl}$ : Gly $=1: 2$ molar ratio) in different mass ratios. 

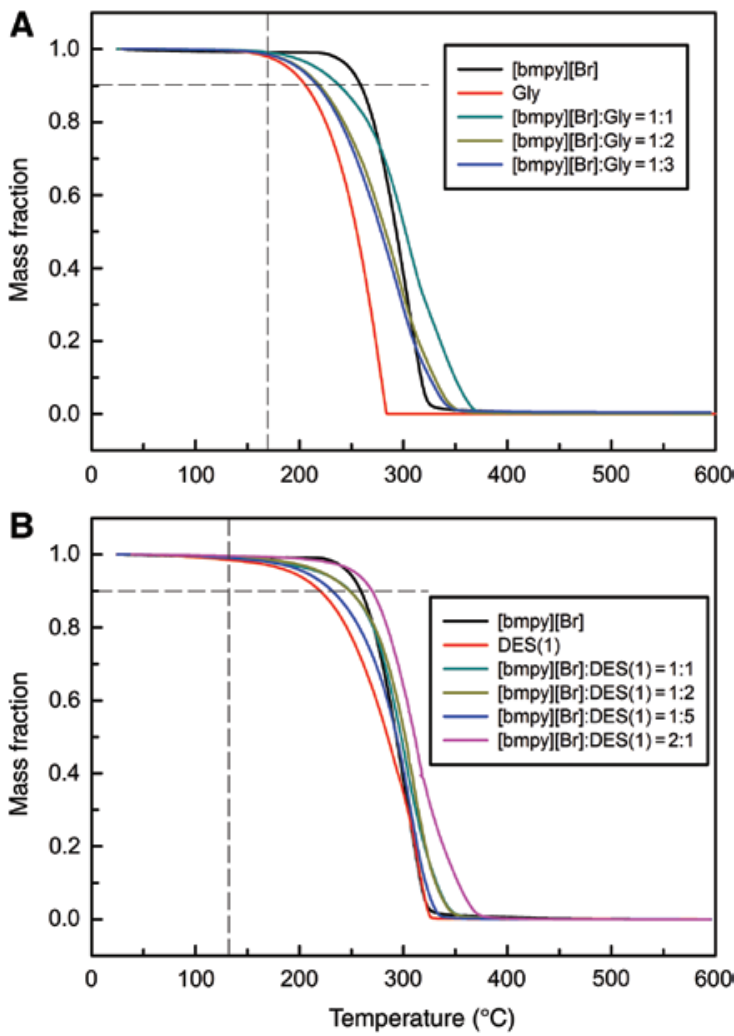

Figure 3: TGA curves of (A) BDES and single components and (B) TDES and single components. The BDES are composed of [bmpy][Br] and glycerol in different molar ratios, and the TDES is composed of [bmpy][Br] and DES(1) (ChCl:Gly=1:2 molar ratio) in different mass ratios.

decreased from mass fraction of 1.0 to 0 . Generally, the prepared DESs are stable up to nearly $200^{\circ} \mathrm{C}$, which is similar to some published study [44]. The $10 \%$ weight loss temperature of each sample is summarized and shown in Figure 4. With the increase of the $[\mathrm{bmpy}][\mathrm{Br}]$ molar fraction, the

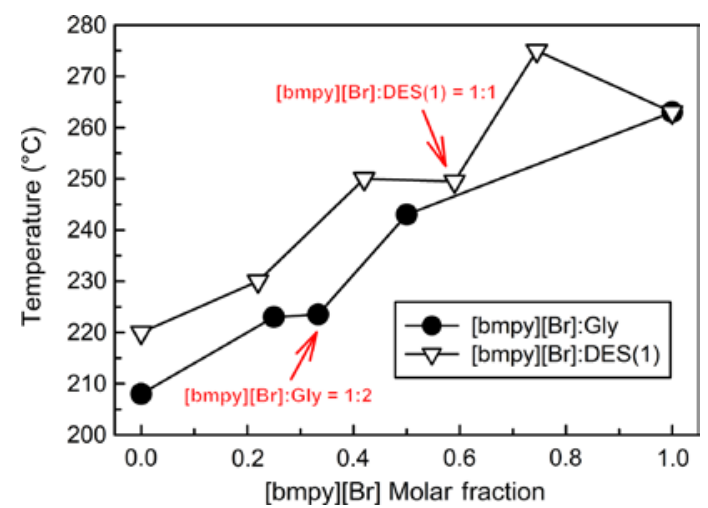

Figure 4: The $10 \%$ weight loss temperature obtained from the TGA for BDES and TDES versus [bmpy][Br] molar fraction. Error bars are smaller than the data points in the graph.
$10 \%$ weight loss temperature of BDES or TDES increases. The higher the $10 \%$ weight loss temperature is, the better thermal stability the sample has. Therefore, the addition of the [bmpy][Br] component is helpful to increase the thermal stability of either BDES or TDES. At similar [bmpy] [Br] molar fraction, the TDES has higher $10 \%$ weight loss temperature than the BDES. It is indicated that the TDES has better thermal stability at $10 \%$ weight loss temperature. For BDES, the $10 \%$ weight loss temperature kept increasing with the increase of [bmpy][Br] molar ratio, and increased slowly when it was around the point of [bmpy] $[\mathrm{Br}]: \mathrm{Gly}=1: 2$ (molar ratio). For TDES, the 10\% weight loss temperature also showed a trend of increase with the increase of [bmpy] $[\mathrm{Br}]$ mass ratio, and also a slower increasing rate appeared, which is at the point of [bmpy][Br]:DES(1)=1:1 (mass ratio). For common molecular hydrogen bonding substances, the strength of the hydrogen bonds decreases with increasing temperature [45]. It is proposed that the slower decrease at the certain ratios was due to the largest content of eutectic mixture formation with the highest hydrogen bond strength. The highest $10 \%$ weight loss temperature for the BDES appeared at the highest [bmpy][Br] ratio. However, the highest $10 \%$ weight loss temperature for the TDES appeared at the [bmpy][Br]:DES(1) = 2:1 (mass ratio), which is higher than either the single components or other DES. This is not a common phenomenon, because not all of the DESs showed higher thermal stability compared to their single compositions, which is usually dependent on the original thermal stability of the starting materials [5]. It is indicated that the addition of [bmpy][Br] can dramatically increase the thermal stability of TDES to a $10 \%$ weight loss temperature higher than any single component. Overall, TDES has slower weight loss rate than BDES with the increase of temperature, thus has better thermal stability.

\subsection{Viscosity}

The dynamic viscosities $(\eta)$ of BDES and TDES were measured in the range of $5^{\circ} \mathrm{C}-80^{\circ} \mathrm{C}$ with an interval of $5^{\circ} \mathrm{C}$. The current spindle could accurately measure $(0.2-6000 \mathrm{cP})$, so the numbers read from the instrument showed large instability when out of range. Therefore, only the viscosity data within the spindle range and stability within $\pm 1 \%$ were taken for analysis. With the increase of temperature, the viscosities decreased. For all of the measured viscosities of DESs near room temperature (Table S1-Table S7 in Supplementary Material), the data were smaller than $500 \mathrm{cP}$, which is much smaller than the viscosity of Gly ( $-950 \mathrm{cP})$. The lowered viscosity of the mixtures compared to the starting composition is another feature 
of eutectic mixtures [46]. The viscosities of temperature dependence were fitted to the logarithmic form of the Arrhenius model with the assumption that the fluid flow obeys the Arrhenius equation for molecular kinetics, and many ILs and DES(s) were also proved to follow this model $[47,48]$.

$$
\ln (\eta)=\ln \left(\eta_{0}\right)+\frac{E_{\eta}}{R T}
$$

where $\eta$ is the dynamic viscosity, $\eta_{0}$ is the coefficient, $E_{\eta}$ is the activation energy for viscous flow, $R$ is the universal gas constant and $T$ is the temperature in $\mathrm{K}$. Based on this equation, the logarithm of the dynamic viscosity is linear to $T^{-1}$, and the activation energy could also be calculated based on the fitting (Figure 5), which is shown in Table 2. The activation energy in the model may differ from different physical sizes, entanglement, or strong interionic interactions [49]. From this table, it is seen that the activation energy of TDES is smaller than that in BDES. It is indicated that the energy barrier that the ions must overcome to move in TDES is smaller than that in BDES.
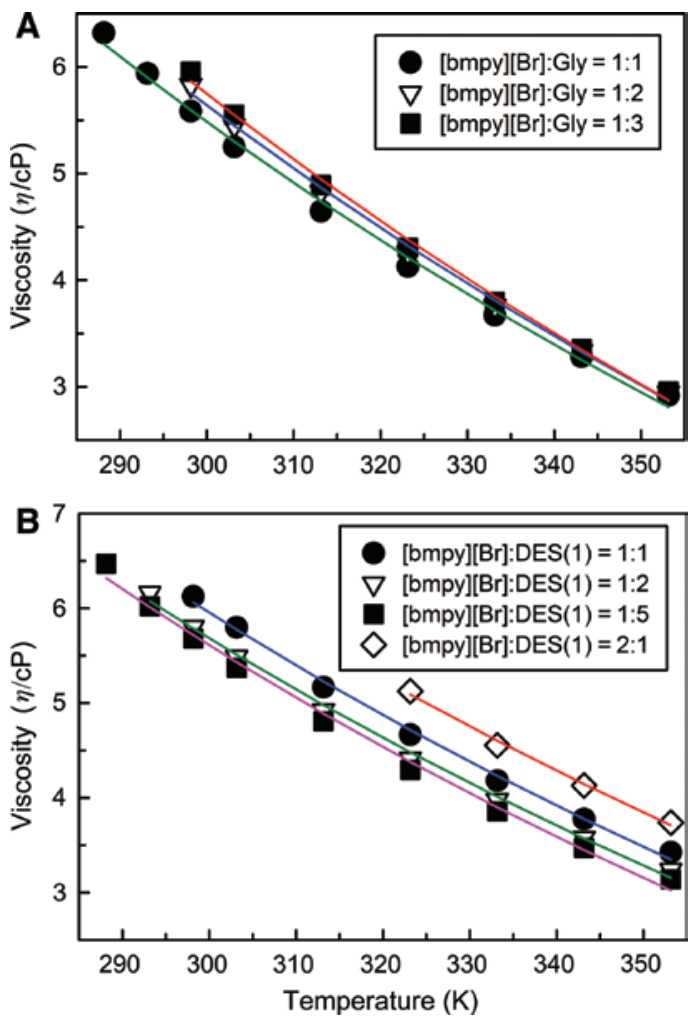

Figure 5: Viscosity in logarithm form versus temperature plots of $(A)$ BDES and (B) TDES. The data points are viscosity data obtained from experiment (error bars are smaller than data points), and the solid lines are fittings of the data to Arrhenius model.
Table 2: Fitting parameters of the viscosity data to Arrhenius model.

\begin{tabular}{lrrr}
\hline DES ratio $^{\mathrm{a}}$ & $\boldsymbol{\eta}_{\mathbf{0}}$ & $\boldsymbol{r}^{\mathbf{2}}$ & $\boldsymbol{E}_{\boldsymbol{\eta}}(\mathbf{2 9 8} \mathbf{K}) / \mathbf{k J ~ m o l}^{-1}$ \\
\hline [bmpy][Br]:Gly=1:1 & 4.61 & 0.9962 & 44.33 \\
[bmpy][Br]:Gly=1:2 & 2.89 & 0.9972 & 45.88 \\
[bmpy][Br]:Gly=1:3 & 1.61 & 0.9966 & 47.62 \\
[bmpy][Br]:DES(1)=2:1 & 14.46 & 0.9958 & 43.62 \\
[bmpy][Br]:DES(1)=1:1 & 11.57 & 0.9970 & 43.21 \\
[bmpy][Br]:DES(1)=1:2 & 14.81 & 0.9970 & 41.92 \\
[bmpy][Br]:DES(1)=1:5 & 9.33 & 0.9947 & 42.88 \\
\hline
\end{tabular}

The table summarizes the associated parameters according to the Arrhenius model. $\eta_{0}$ is a coefficient developed from the interception of the linearly fitting, and $E_{\eta}$ is the activation energy developed from the slope of the linearly fitting.

aThe BDES is composed of [bmpy] $[\mathrm{Br}]$ and glycerol in different molar ratios. The TDES is composed of [bmpy] $[\mathrm{Br}]$ and DES(1) ( $\mathrm{ChCl}: \mathrm{Gly}=1: 2$ molar ratio) in different mass ratios.

\subsection{Density}

The density data for [bmpy][Br]:DES(1)=2:1 mass ratio was not collected, because the high viscosity of the mixture leads to difficulty in the liquid transfer during the gravity bottle measurement. The density data for other mixtures were collected at room temperature $\left(22^{\circ} \mathrm{C}\right)$ and plotted in Figure 6. For all of the tested DESs, the density lied in the range of $1.27-1.33 \mathrm{~g} / \mathrm{cm}^{3}$. In a study by Shahbaz et al. [50], it was concluded that the density of the DESs lies between the densities of the corresponding salt and the hydrogen bond donor. In our case, the density of the prepared BDES and TDES was between Gly $\left(\sim 1.26 \mathrm{~g} / \mathrm{cm}^{3}\right)$ and the solids, so our results are consistent with their conclusion. For both BDES and TDES, the density and the [bmpy][Br] molar fraction have linear relationship $\left(r^{2} \geq 0.9\right)$. However, with the increase of [bmpy][Br] molar fraction, BDES and TDES

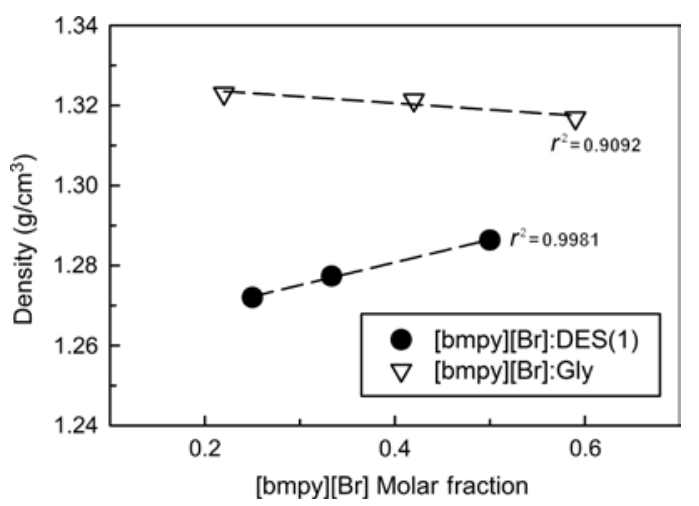

Figure 6: Densities of the binary and ternary mixtures based on [bmpy] [Br] molar fraction. Error bars are smaller than the data points in the graph. 
displayed different trend. Overall, the density of the TDES is smaller than that of BDES. From the current density data, the free volume in the TDES is larger than that of BDES, because the density of the TDES is smaller. The large free volume may come from the inhomogeneity of the ions/molecules. In this experiment, there are two kinds of ions and one kind of molecule in the BDES, but there are four kinds of ions and one kind of molecule in the TDES. It is proposed that the inhomogeneity of the ions/molecules leads to different particle sizes and movement directions, and further creates more free volume between each other.

\subsection{Future work and applications}

As novel DESs were prepared in this study, future work may include characterizations of more properties (e.g. refractive index, ionic conductivity, surface tension) to further investigate the new material. From the previous publication, Abbott developed the hole theory [29] for the design of improved DES, which discussed the utilization of the free volume of a liquid. From this point, there is free volume in either BDES or TDES. The prepared TDESs have large free volume, which make it capable for gas sorption or gas storage. The high thermal stability provides better guarantee for the potential high temperature applications.

\section{Conclusions}

In this work, BDES and TDES prepared with [bmpy][Br] were prepared and their physiochemical properties were characterized. The preparation process was green and highly efficient. From the DSC results, no melting peak was observed for the prepared mixtures, and only glass transition temperature $\left(T_{\mathrm{g}}\right)$ at around $-25^{\circ} \mathrm{C}$ was observed. The viscosities of the prepared mixtures were also lower than the starting compositions. The lack of the peak events in DSC and lowered viscosity of the mixtures could serve as the evidence of eutectic mixtures formation. The TGA results indicated that the TDES had better thermal stability than the BDES overall. Lower energy barrier for the ions to move and smaller density were also obtained for TDES. It is proposed that more ions/ molecules in the liquid system create more inhomogeneity leading to more free volume in the TDES. The free volume is potentially useful for sorption applications, and the prepared TDES is also suitable for high thermal requirement applications.

\section{References}

[1] Baker GA, Baker SN, Pandey S, Bright FV. Analyst 2005, 130, 800-808.

[2] de María PD, Maugeri Z. Curr. Opin. Chem. Biol. 2011, 15, 220-225.

[3] Plechkova NV, Seddon KR. Chem. Soc. Rev. 2008, 37, 123-150.

[4] Abbott AP, Boothby D, Capper G, Davies DL, Rasheed RK. J. Am. Chem. Soc. 2004, 126, 9142-9147.

[5] Francisco M, van den Bruinhorst A, Kroon MC. Angew. Chem. Int. Ed. 2013, 52, 3074-3085.

[6] Tang B, Row KH. Monatsh. Chem. 2013, 144, 1427-1454.

[7] Smith EL, Abbott AP, Ryder KS. Chem. Rev. 2014, 114, 11060-11082.

[8] María PD, Maugeri Z. Curr. Opin. Chem. Biol. 2011, 15, 220-225.

[9] Abbott AP, Cullis PM, Gibson M], Harris RC, Raven E. Green Chem. 2007, 9, 868-872.

[10] Arain MB, Yilmaz E, Soylak M. J. Mol. Liq. 2016, 224, 538-543.

[11] Li X, Row KH. J. Sep. Sci. 2016, 39, 3505-3520.

[12] Warrag SE, Peters CJ, Kroon MC. Curr. Opin. Green Sustain. Chem. 2017, 5, 55-60.

[13] Liao HG, Jiang YX, Zhou ZY, Chen SP, Sun SG. Angew. Chem. 2008, 120, 9240-9243.

[14] Kumaraguru S, Pavulraj R, Vijayakumar J, Mohan S. J. Alloys Comp. 2017, 693, 1143-1149.

[15] Mota-Morales JD, Gutiérrez MC, Ferrer ML, Sanchez IC, Elizalde-Peña EA, Pojman JA, Del Monte F, Luna-Bárcenas G. J. Polym. Sci. Part A Polym. Chem. 2013, 51, 1767-1773.

[16] Carriazo D, Serrano MC, Gutiérrez MC, Ferrer ML, del Monte F. Chem. Soc. Rev. 2012, 41, 4996-5014.

[17] Francisco M, van den Bruinhorst A, Kroon MC. Green Chem. 2012, 14, 2153-2157.

[18] Gorke J, Srienc F, Kazlauskas R. Biotechnol. Bioprocess Eng. 2010, 15, 40-53.

[19] Mbous YP, Hayyan M, Hayyan A, Wong WF, Hashim MA, Looi CY. Biotechnol. Adv. 2016, 35, 105-134.

[20] Ruß C, König B. Green Chem. 2012, 14, 2969-2982.

[21] Khandelwal S, Tailor YK, Kumar M. J. Mol. Liq. 2016, 215, 345-386.

[22] Alonso DA, Baeza A, Chinchilla R, Guillena G, Pastor IM, Ramón DJ. Eur. J. Org. Chem. 2016, 2016, 612-632.

[23] Kumar V, Nigam KDP. Green Process Synth. 2012, 1, 79-107.

[24] Durand E, Lecomte J, Villeneuve P. Biochimie 2016, 120, 119-123.

[25] Taysun MB, Sert E, Atalay FS. J. Mol. Liq. 2016, 223, 845-852.

[26] Martins MA, Paveglio GC, Munchen TS, Meyer AR, Moreira DN, Rodrigues LV, Frizzo CP, Zanatta N, Bonacorso HG, Melo PA. J. Mol. Liq. 2016, 223, 934-938.

[27] Craveiro R, Aroso I, Flammia V, Carvalho T, Viciosa M, Dionísio M, Barreiros S, Reis R, Duarte ARC, Paiva A. J. Mol. Liq. 2016, 215, 534-540.

[28] Espino M, de los Ángeles Fernández M, Gomez FJ, Silva MF. TrAC Trends Anal. Chem. 2016, 76, 126-136.

[29] Abbott AP, Capper G, Gray S. Chem. Phys. Chem. 2006, 7, 803-806.

[30] Sze LL, Pandey S, Ravula S, Pandey S, Zhao H, Baker GA, Baker SN. ACS Sust. Chem. Eng. 2014, 2, 2117-2123.

[31] Chemat F, Anjum H, Shariff AM, Kumar P, Murugesan T. J. Mol. Liq. 2016, 218, 301-308.

[32] Liu YT, Chen YA, Xing YJ. Chin. Chem. Lett. 2014, 25, 104-106. 
[33] Jhong HR, Shan D, Wong H, Wan CC, Wang YY, Wei TC. Electrochem. Commun. 2009, 11, 209-211.

[34] Abdel-Fattah TM, Loftis JD, Mahapatro A. Nanosci. Nanotechnol. 2015, 5, 36-44.

[35] Rogers RD, Voth GA. Acc. Chem. Res. 2007, 40, 1077-1078.

[36] Burrell AK, Sesto RED, Baker SN, McCleskey TM, Baker GA. Green Chem. 2007, 9, 449-454.

[37] Tagliapietra S, Orio L, Palmisano G, Penoni A, Cravotto G. Chem. Papers 2015, 69, 1519-1531.

[38] Abbott AP, Harris RC, Ryder KS, D’Agostino C, Gladden LF, Mantle MD. Green Chem. 2011, 13, 82-90.

[39] Petkovic M, Seddon KR, Rebelo LPN, Pereira CS. Chem. Soc. Rev. 2011, 40, 1383-1403.

[40] Abbott AP, Harris RC, Ryder KS, D'Agostino C, Gladdenb LF, Mantle MD. Green Chem. 2011, 13, 82-90.

[41] Morrison HG, Sun CC, Neervannan S. Int. J. Pharm. 2009, 378, 136-139.

[42] Abbott AP, Capper G, Davies DL, Rasheed RK, Tambyrajah V. Chem. Commun. 2003, 70-71.

[43] Francisco M, van den Bruinhorst A, Zubeir LF, Peters C], Kroon MC. Fluid Phase Equilibria. 2013, 340, 77-84.

[44] Zhao H, Baker GA, Holmes S. J. Mol. Catal. B Enzy. 2011, 72, 163-167.

[45] Dougherty RC. J. Chem. Phys. 1998, 109, 7372-7378.

[46] Yadav A, Pandey S. J. Chem. Eng. Data 2014, 59, 2221-2229.

[47] Mahurin SM, Yeary JS, Baker SN, Jiang DE, Dai S, Baker GA. J. Mem. Sci. 2012, 401-402, 61-67.

[48] Bahadori L, Chakrabarti MH, Mjalli FS, AINashef IM, Manan NSA, Hashim MA. Electrochim. Acta 2013, 113, 205-211.

[49] Pandey S, Baker GA, Sze L, Pandey S, Kamath G, Zhao H, Baker SN. New J. Chem. 2013, 37, 909-919.

[50] Shahbaz K, Mjalli F, Hashim M, AlNashef I. Thermochim. Acta 2011, 515, 67-72.

Supplementary Material: The online version of this article offers supplementary material (https://doi.org/10.1515/gps-2017-0060).

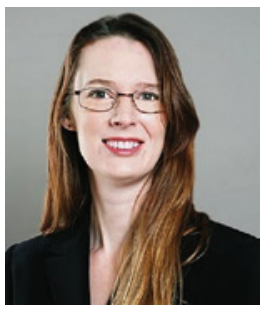

Sheila N. Baker

Sheila N. Baker obtained her BS in Chemistry in 1997 at Georgia Southern University, and then her PhD in 2002 (advisor: F. V. Bright) at the University at Buffalo, The State University of New York. After staying as a postdoctoral Associate at Los Alamos National Laboratory (T. M. McCleskey) and Oak Ridge National Laboratory, she joined University of Missouri, Columbia as an Assistant Professor in 2011. Her primary research interests include sub- and supercritical fluids, designer ILs and deep eutectic solvents, magnetic and morphologically unique nanostructures, and materials for nextgeneration photovoltaics, batteries, and supercapacitors.

\section{Bionotes}

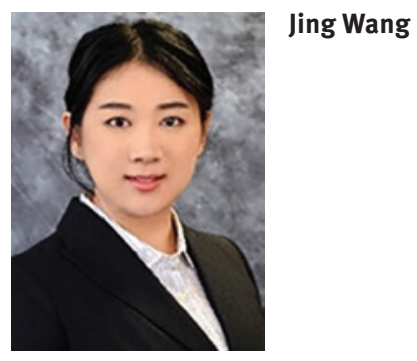

Jing Wang received her BS in Chemical Engineering at the East China University of Science and Technology in Shanghai, China in 2012. In 2015, she received her PhD in Chemical Engineering (advisor: Sheila Baker) at the University of Missouri, Columbia, MO, USA. She has been a postdoctoral fellow (Qingsong Yu and $\mathrm{Hao} \mathrm{Li)} \mathrm{at} \mathrm{the} \mathrm{same}$ university after graduation. Her research interests are ionic liquid (IL) and deep eutectic solvent related functional materials, biomaterials, and environmental friendly materials. 